\title{
Economic Hardships and Self-reported Deterioration of Physical and Mental Health Under the COVID-19 Pandemic: A Cross-sectional Study, 2020, Japan
}

\author{
Satomi Odani ${ }^{1}$, Tomohiro Shinozaki ${ }^{2}$, Kenji Shibuya ${ }^{3,4}$, and Takahiro Tabuchi ${ }^{1,4}$ \\ ${ }^{1}$ Cancer Control Center, Osaka International Cancer Institute, Osaka, Japan \\ ${ }^{2}$ Tokyo University of Science, Department of Information and Computer Technology, Tokyo, Japan \\ ${ }^{3}$ Soma COVID Vaccination Medical Center, Fukushima, Japan \\ ${ }^{4}$ The Tokyo Foundation for Policy Research, Tokyo, Japan \\ Received June 2, 2021; accepted January 17, 2022; released online January 29, 2022
}

\begin{abstract}
Background: Coronavirus disease 2019 (COVID-19) has disproportionately affected the most vulnerable populations. We assessed the prevalence and disparities of economic hardships and their impact on health deterioration in Japan.

Methods: Data were obtained from a nation-wide, cross-sectional, internet-based, self-reported survey conducted during August-September, 2020 with individuals aged $15-79$ years in Japan $(n=25,482)$. Economic hardships and changes in various physical and mental health status were measured using sample-weighted data. Adjusted prevalence ratios (APRs) were estimated to investigate the associations between economic hardships and health outcomes.

Results: During April-September, 2020 in Japan, 25.0\%, 9.6\%, 7.9\%, and 3.1\% of the respondents experienced income loss, money shortage, financial anxiety and financial exploitation, respectively, with higher prevalence among workers (vs nonworkers). Stratifying by sex and working status, income loss was associated with physical health deterioration (APRs ranged from 1.45-1.95), mental health deterioration (APRs ranged from 1.47-1.68), and having serious psychological distress (APRs ranged from 1.41-2.01) across all strata. Shortage of money and financial anxiety were also associated with increased likelihood of all adverse health outcomes assessed, regardless of whether the hardships were pre-existing or experienced first time. Among non-working individuals, financial exploitation was associated with physical health deterioration among males (APR 1.88) and mental health deterioration among both males (APR 1.80) and females (APR 2.23), while such associations were not observed among working individuals.

Conclusions: During the early phase of the COVID-19 epidemic, COVID-19-related economic hardships were associated with physical and mental health deterioration in Japan, particularly among the vulnerable populations. Timely and prompt responses are warranted to mitigate both economic and health burdens.
\end{abstract}

Key words: mental health; self-rated health; health disparities; social determinants of health

Copyright $\odot 2022$ Satomi Odani et al. This is an open access article distributed under the terms of Creative Commons Attribution License, which permits unrestricted use, distribution, and reproduction in any medium, provided the original author and source are credited.

\section{INTRODUCTION}

The novel coronavirus disease 2019 (COVID-19) has posed a devastating socioeconomic and health burden on the society. Many countries have implemented public health responses, including lockdown, border control, mobility restriction, and suspension of businesses. Although they are effective to minimize infection, sudden standstill of the society has resulted in tremendous economic strain that has jeopardized the well-being of the public. ${ }^{1}$

COVID-19 has disproportionately affected the most vulnerable populations $^{2-4}$; racial/ethnic minority, low-income, and young individuals have faced disproportionately high rates of job loss and income loss in many countries. ${ }^{4-6}$ Such economic hardships may lead to shortage of resources for housing, food, and medical and essential services, underscoring the urgent need to develop robust safety net programs to help those who experience the greatest harms. To support the livelihood of people under the COVID-19 pandemic, many countries and regions, such as the United States, Republic of Korea, and Hong Kong, introduced financial relief programs. ${ }^{7-9}$ The Japanese government also launched the Special Cash Payments program in April 2020 to provide one-time, across-the-board $¥ 100,000$ (\$930 in USD) relief. ${ }^{10,11}$ While a recent study has implied protective effects of the relief program for the health of workers, ${ }^{12}$ whether the relief has reached the individuals who bear the heaviest burden, including non-working individuals, remains understudied.

There also is a concern that the COVID-19-induced economic turmoil would widen the pre-existing health inequalities. ${ }^{6,13,14}$ An assessment of workers in six European countries demonstrates 
that economic hardships, such as income loss, job loss, and workload decrease during the pandemic were associated with increased mental health complaints. ${ }^{15}$ This relationship was more apparent among workers in a lower social gradient. Several other studies of working populations support this finding ${ }^{16-18}$; there exists a knowledge gap as to whether this is generalizable to other domains of health and other countries with diverse COVID-19 impact and different social systems. Filling this knowledge gap will enable us to provide important implications to design future interventions to mitigate health and social impacts of COVID-19, especially among the most vulnerable populations.

The aim of this study is to assess the levels and distribution of prevalence in economic hardships during the COVID-19 pandemic and their impact on health status. We examined three key components of economic hardship (income poverty, material deprivation, and subjective financial stress $)^{19,20}$ along with two indicators of COVID-19-related economic hardships to obtain a more comprehensive understanding of personal or interpersonal experiences. We investigated the effects of both pre-existing economic hardships and those instantaneously induced during the COVID-19 pandemic. We additionally conducted stratified analyses by age, sex, and working status to examine whether the impact of economic hardships differed by these demographics.

\section{METHODS}

\section{Internet survey}

We obtained data from the Japan COVID-19 and Society Internet Survey (JACSIS), a nation-wide, cross-sectional, internet-based, self-reported survey of individuals aged 15 to 79 years. The JACSIS survey was administered by a private vendor, Rakuten Insight Inc., that had nearly 2.2 million qualified panelists in $2019 .^{21}$ At the time of registration, the panelists were asked to provide their demographic information and a web-based informed consent. The questionnaires were distributed to 224,389 panelists selected by sex, age, and residing prefecture using simple random sampling. Data collection started on August $25^{\text {th }}$ and closed on September $30^{\text {th }}, 2020$, when the target sample size of 28,000 was met (response rate, $12.5 \%$ ). There were no missing responses as all questions were required to be answered. We excluded 2,518 individuals who provided unnatural or inconsistent responses using the algorithm we developed, ${ }^{22}$ which resulted in the final sample size of 25,482 individuals. The study was reviewed and approved by the Research Ethics Committee of the Osaka International Cancer Institute (no. 20094-2).

\section{Variables}

\section{Economic hardships (exposure variables)}

To assess the three major components of economic hardship (income poverty, material deprivation, and subjective financial stress), ${ }^{19,20,23}$ we utilized the following indicators that correspond to each of the components: income loss, shortage of money to buy things or pay bills, and anxiety about household budget (financial anxiety). Income loss was assessed with the question "When your income before the COVID-19 pandemic is taken as 100 , how would you rate your income now? If your income has decreased by half, please rate as 50 ; if increased to double, please rate as 200." Rating under 100 was defined as income loss. Shortage of money was assessed by asking respondents whether they had experienced shortage of money to buy or pay for items, such as rent/mortgage, food, medical/dental fees, tuition, and other necessities during April-September, 2020. Financial anxiety was assessed by asking respondents whether they had felt anxious about their household budget during AprilSeptember, 2020. For money shortage and financial anxiety, respondents were also asked whether they had experienced the hardships continuously before the COVID-19 pandemic or experienced first time since the pandemic took place. We considered the latter as a proxy of the COVID-19-induced economic hardship for descriptive analyses, while both pre-existing hardships and those instantaneously arose during the pandemic were employed to investigate their effects on health.

We further assessed two relevant measures of economic hardship, financial exploitation and non-receipt of the across-theboard relief, to obtain a nuanced understanding of personal or interpersonal experiences during the pandemic among various populations. Financial exploitation was assessed by asking respondents whether their saving or pension was used or taken by someone, including their family, without their permission during April-September, 2020. Non-receipt of the cash relief was assessed by asking respondents whether they had received the Special Cash Payment (\$930 relief) during the COVID-19 pandemic by the time of data collection (April-September, 2020). Deterioration and current state of physical/mental health (outcome variables)

As we were particularly interested in changes in health status during the COVID-19 pandemic, we assessed self-reported deterioration of physical and mental health as the primary outcomes. Respondents were asked "How does your current physical/mental health state compare to that in or before January 2020?" in separate questions. Responses "Worsened very much/ somewhat" were defined as health deterioration (vs "Improved very much/somewhat", "Unchanged" and "I don't know").

To provide a cross-sectional landscape of the health of the public under the COVID-19 pandemic, we also assessed two measures of current health status as the secondary outcomes. Respondents were asked to rate their general health status using a 5-point Likert scale. Responses were dichotomized as to be favorable (excellent/very good/good) or unfavorable (poor/fair). Presence of serious psychological distress (SPD) in the past 30 days was also assessed using the Kessler 6 (K6) scale: K6 scores $\geq 13$ were defined as having SPD. ${ }^{24}$

\section{Respondents' characteristics (confounders)}

Assessed characteristics included sex; age; education; past-year income level, classified using the latest national baseline income (ie, poverty line) ${ }^{25}$; marital status; employment status; and the number of COVID-19 cases in the residing area (cumulative number of cases during January $1^{\text {st }}$-August $25^{\text {th }}, 2020$, of each prefecture was divided into tertiles and used to classify areas of respondents' residence). ${ }^{26}$ History of physical morbidities (cancer or malignant tumor, diabetes, asthma, bronchitis, chronic obstructive pulmonary disease, angina, myocardial infarction, and/or cerebral infarction) and mental illness (depression or any other mental disorder) was also assessed.

\section{Statistical analysis}

To account for the potential selection bias of the internet-based sample, data were weighted using inverse probability weighting (IPW). We used a nationally representative population-based sample from the Comprehensive Survey of Living Conditions of People on Health and Welfare (CSLCPHW). ${ }^{27}$ Data from the two 
surveys (JACSIS and CSLCPHW) were combined and used for a logistic regression accounting for basic demographic characteristics such as sex, age, region, marital status, education, employment, and health status to estimate the probability of "being a respondent in the internet survey." Further details regarding IPW are reported elsewhere. ${ }^{22,28}$

Adjusted prevalence ratios (APRs) were estimated through weighted multivariable-adjusted log-linear models fitted by the least-square method followed by robust variance estimation (also known as "modified Poisson" regression) to account for IPW and circumvent the specification of outcome distributions. First, we modeled the prevalence of economic hardships and health outcome measures as separate outcome (dependent) variables to investigate their possible predictors. We further compared the prevalence of health outcomes between presence versus absence of economic hardships. We fitted separate models for each of the combinations of an economic hardship variable and a health outcome variable within each of the population subgroups stratified by sex, age, and working status (working vs non-working). All analyses were conducted using $\mathrm{R}$ version 3.4.1 (R Foundation for Statistical Computing, Vienna, Austria).

\section{RESULTS}

Economic hardships during the COVID-19 pandemic Demographic and socioeconomic characteristics of respondents are presented in eTable 1. Weighted analyses showed that, among all respondents, the prevalence of economic hardships during the COVID-19 pandemic was $25.0 \%$ (income loss), $9.6 \%$ (money shortage), $7.9 \%$ (financial exploitation), $3.1 \%$ (financial exploitation), and $13.6 \%$ (non-receipt of the relief) (Table 1). Working individuals had higher prevalence of income loss, money shortage, financial anxiety, and financial exploitation among both males and females (eTable 2 and eTable 3 ).

While females were more likely to report income loss (APR 1.28; 95\% confidence interval [CI], 1.14-1.45) and financial anxiety (APR 2.02; 95\% CI, 1.56-2.62), they were less likely to report financial exploitation (APR 0.45; 95\% CI, 0.32-0.64) and non-receipt of the relief (APR 0.58; 95\% CI, 0.46-0.74) compared to males (Table 1). Younger individuals were more likely to experience the assessed economic hardships, except income loss: the strongest association was seen for financial exploitation among individuals aged 25-34 years (APR 16.04; [95\% CI, 9.08-28.35 vs the oldest group). Financial exploitation was also more likely among individuals with lower education (APR 1.60; 95\% CI, 1.14-2.25 and APR 1.52; 95\% CI, 1.04-2.21 among those with $\leq$ high school education and some college education vs college graduates) and those with past or present physical chronic disease.

Compared to the most affluent group, individuals whose household past-year income was below the baseline had 1.71 (95\% CI, 1.19-2.45), 1.46 (95\% CI, 1.07-1.98), and 1.61 (95\% CI, 1.24-2.08) times higher likelihood of experiencing money shortage, financial anxiety, and non-receipt of the relief, respectively. Divorced/widowed individuals were more likely to experience money shortage (APR 1.72; 95\% CI, 1.11-2.67), financial exploitation (APR 1.80; 95\% CI, 1.14-2.84) and nonreceipt of the relief (APR 2.32; 95\% CI, 1.72-3.14), while they had lower likelihood of experiencing income loss (APR 0.58; 95\% CI, 0.41-0.82) and financial anxiety (APR 0.41; 95\% CI, 0.28-0.61) compared to married individuals.
With full-time employees as the referent, employment status was significantly associated with income loss (APR 2.08; 95\% CI, 1.70-2.54 among self-employed individuals), money shortage (APR 1.48; 95\% CI, 1.01-2.15 among part-time workers/contractors), and financial anxiety (APR 1.86; 95\% CI, 1.16-2.98 among self-employed individuals) during the COVID-19 pandemic. Respondents living in the areas with a higher number of COVID-19 infections were more likely to report non-receipt of the relief (APR 1.27; 95\% CI, 1.07-1.51).

\section{Deterioration of physical/mental health and current state of health}

Among all respondents, $15.5 \%$ and $18.5 \%$ reported their physical and mental health had worsened during the COVID-19 pandemic, respectively (Table 2). Current health status was rated as unfavorable by $13.5 \%$ of respondents. Prevalence of SPD was $10.0 \%$, with a wide disparity between male workers and nonworkers (13.9\% vs 3.3\%), while the difference was not as substantial between female workers and non-workers $(9.5 \%$ vs 8.5\%) (eTable 4 and eTable 5).

Females had higher likelihood of mental health deterioration (APR 1.35; 95\% CI, 1.10-1.66) and reporting SPD (APR 1.28; 95\% CI, 1.00-1.65) than males during the COVID-19 pandemic. Younger individuals were more likely to report deterioration of physical and mental health; prevalence of SPD was also higher among young individuals with the strongest association among those aged 15-24 years (APR 5.31; 95\% CI, 2.31-12.18). SPD was also more often reported by individuals whose past-year income was below the baseline (APR 1.49; 95\% CI, 1.04-2.14) and the divorced/widowed (APR 1.55; 95\% CI, 1.01-2.47).

Compared to individuals without a mental illness history, those with past or present mental illness were more likely to report adverse health outcomes, with stronger associations among those currently having the illness (APR 2.58; 95\% CI, 1.84-3.36 and APR 2.30; 95\% CI, 1.68-3.16 for deterioration of physical and mental health, respectively, and APR 3.07; 95\% CI, 2.08-4.53 and APR 5.02; 95\% CI, 3.18-7.90 for unfavorable current health and SPD, respectively).

\section{Associations between economic hardships and physical/mental health during the COVID-19 pan- demic}

After adjusting for confounders, respondents who experienced income loss had 1.45-1.95 times higher likelihood of physical health deterioration and 1.47-1.68 times higher likelihood of mental health deterioration during the COVID-19 pandemic within all sex-work strata (Figure 1). APRs and CIs for overall and each sex-work strata are presented in eTable 6. Money shortage was also a significant predictor of health deterioration, regardless of whether the problem was pre-existing or not: the strongest associations were seen for physical health deterioration among non-working females who experienced money shortage for the first time during the COVID-19 pandemic (APR 2.79; 95\% CI, 2.09-3.72) and for mental health deterioration among nonworking males who experienced money shortage for the first time (APR 2.34; 95\% CI, 1.58-3.46).

Similarly, non-working females and non-working males who reported experiencing financial anxiety for the first time during the COVID-19 pandemic had the highest likelihood of physical health deterioration (APR 3.29; 2.51-4.31) and mental health deterioration (APR 3.53; 95\% CI, 2.46-5.06), respectively. While 


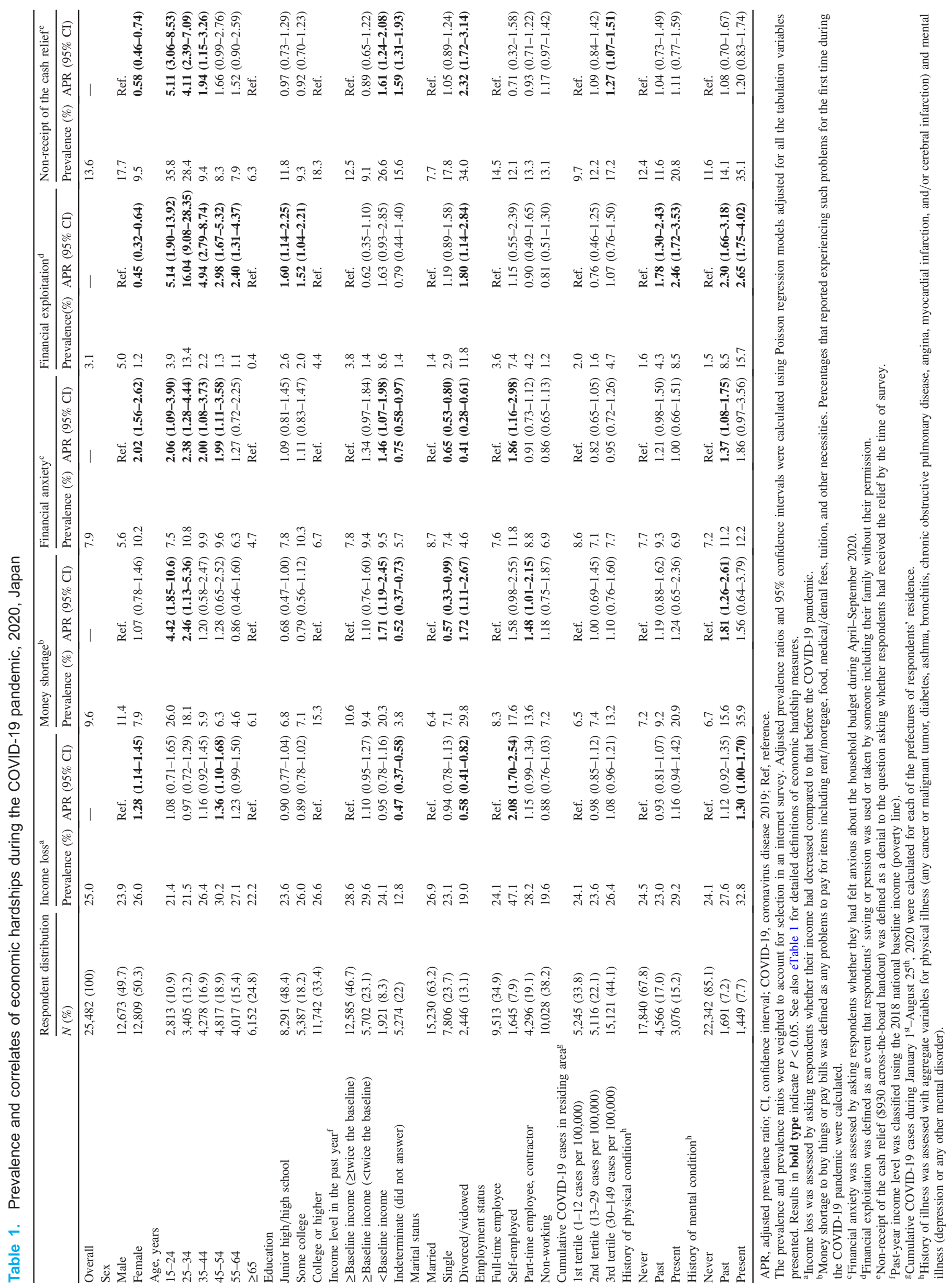




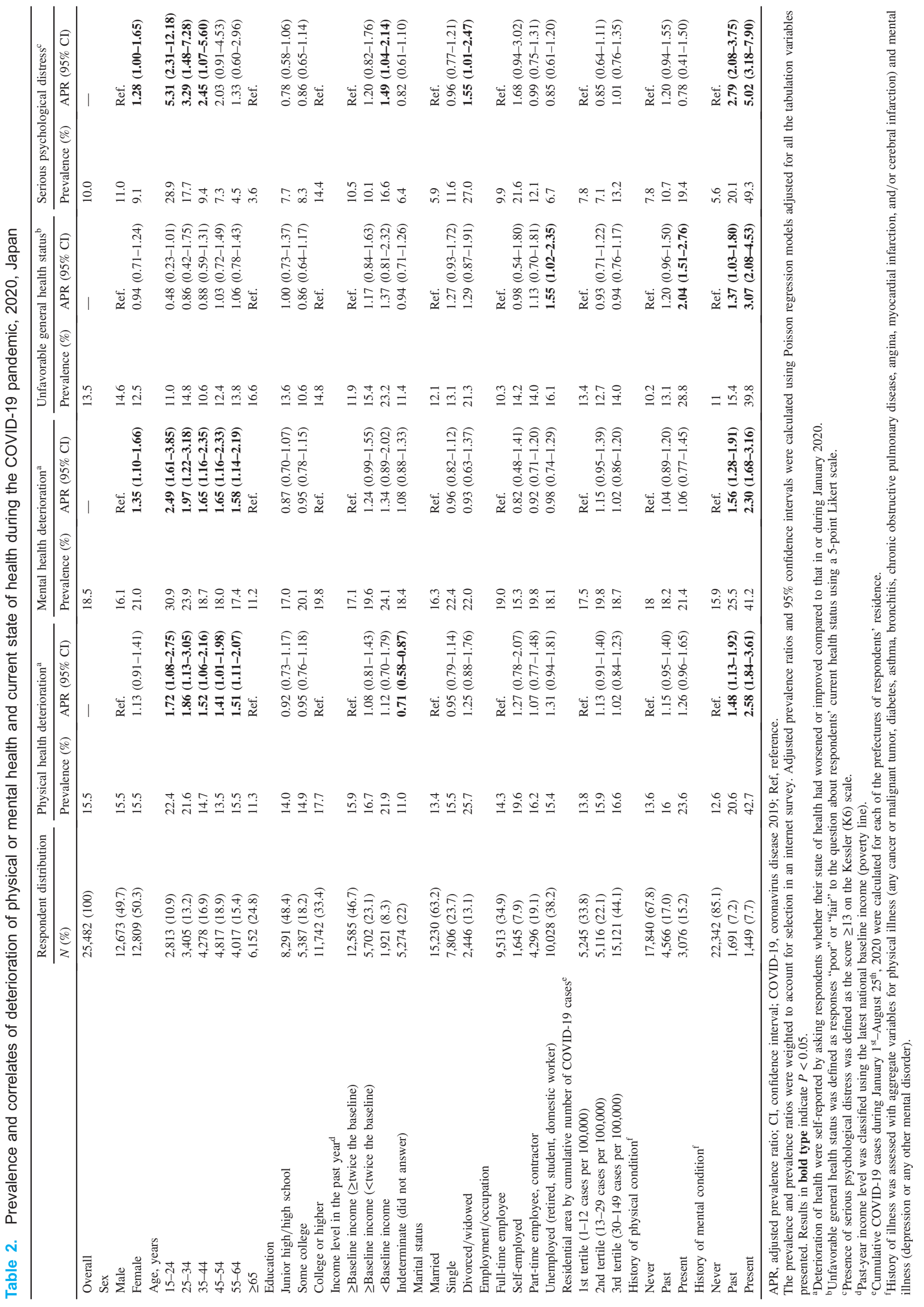



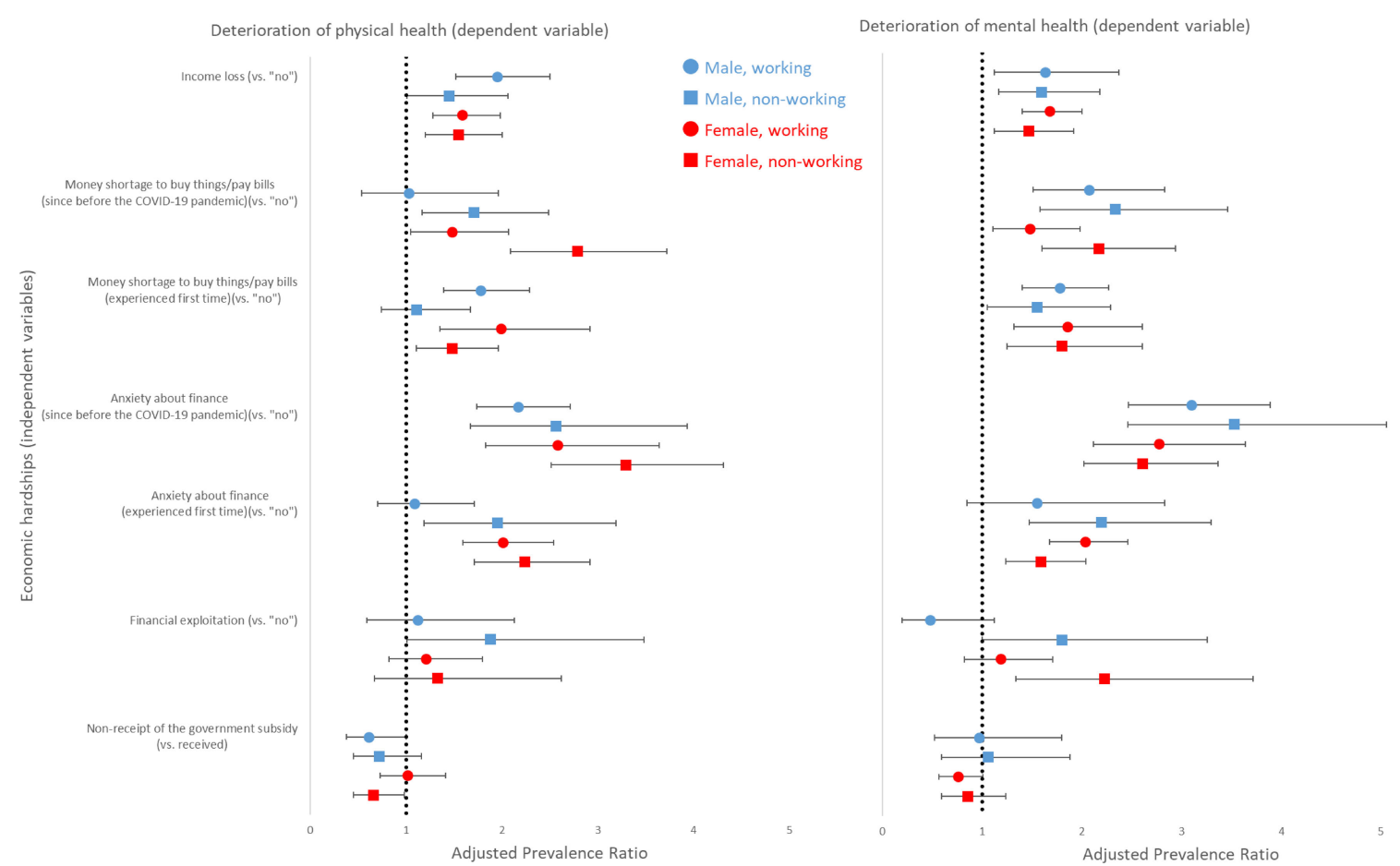

Figure 1. Associations between economic hardships and health deterioration during the COVID-19 pandemic, 2020, Japan. $N=9,008$ (male, working); 3,665 (male, non-working); 6,446 (female, working); 6,363 (female, non-working). Genderand working status-specific adjusted prevalence ratios (95\% confidence intervals) estimated through weighted multivariable-adjusted log-linear models followed by robust variance estimation controlling for age, education, pastyear income level, marital status, cumulative COVID-19 cases in residing area, and history of physical and mental illness. Working status was categorized as to be working (full-time employee, self-employed, part-time/contractor) or non-working (retired, student, domestic worker, unemployed). Deterioration of health was self-reported by asking respondents whether their state of health had worsened compared to that in or during January 2020. COVID-19, coronavirus disease 2019.

financial exploitation was not significantly associated with health deterioration in working populations, it significantly predicted physical health deterioration among non-working males (APR 1.88 ; 95\% CI, 1.01-3.48) and mental health deterioration among non-working males (APR 1.80; 95\% CI, 1.00-3.26) and nonworking females (APR 2.23; 95\% CI, 1.34-3.72). Non-receipt of the cash relief was associated with lower likelihood of physical health deterioration among non-working females (APR 0.66; 0.45-0.99).

Figure 2 and eTable 6 present associations between economic hardships and current state of health. Consistent to the findings in Figure 1, income loss, money shortage, and financial anxiety were significantly associated with unfavorable health condition and presence of SPD within most of sex-work strata. Notably, working males who experienced money shortage for the first time during the COVID-19 pandemic were 5.18 (95\% CI, 3.72-7.21) times more likely to have SPD compared to those who did not report money shortage. Financial anxiety was associated with increased likelihood of both unfavorable current health and SPD, with generally stronger associations in non-working populations. Financial exploitation also predicted the presence of SPD with the strongest association among non-working males (APR 5.04; $95 \%$ CI, 2.53-10.07). eTable 7 presents stratified APRs by age $(<65$ vs $\geq 65$ years) to investigate whether the impact of economic hardships differs for younger adults versus older adults based on the assumption that working status had different implications for these two age groups (ie, older adults were mostly retired and non-working). While the strengths of associations differed, the directions of associations were generally consistent between age groups.

\section{DISCUSSION}

Economic hardships-income loss, shortage of money, and financial anxiety-were independently associated with increased likelihood of physical and mental health deterioration, being in unfavorable health conditions, and having SPD, regardless of whether such economic hardships were pre-existing or arose during the COVID-19 pandemic. Financial exploitation was associated with deterioration of physical and mental health among only non-working individuals. Our findings shed light on the vulnerability of certain populations and underscore the importance of timely and targeted interventions to help mitigate the detrimental impact of economic hardships.

We found that one in four individuals in Japan experienced income loss during the COVID-19 pandemic, with higher likelihood among females: more females reported financial anxiety than males. This can be partially attributable to the facts that more women in Japan serve in the industries, such as service, retail, or travel, which were severely damaged by the COVID-19 pandemic, ${ }^{29}$ and that women faced drastic changes in their employment status and reduced working hours more often than their male counterparts. ${ }^{30}$ Although there were no systematic patterns in disparities in income loss across age and past-year 

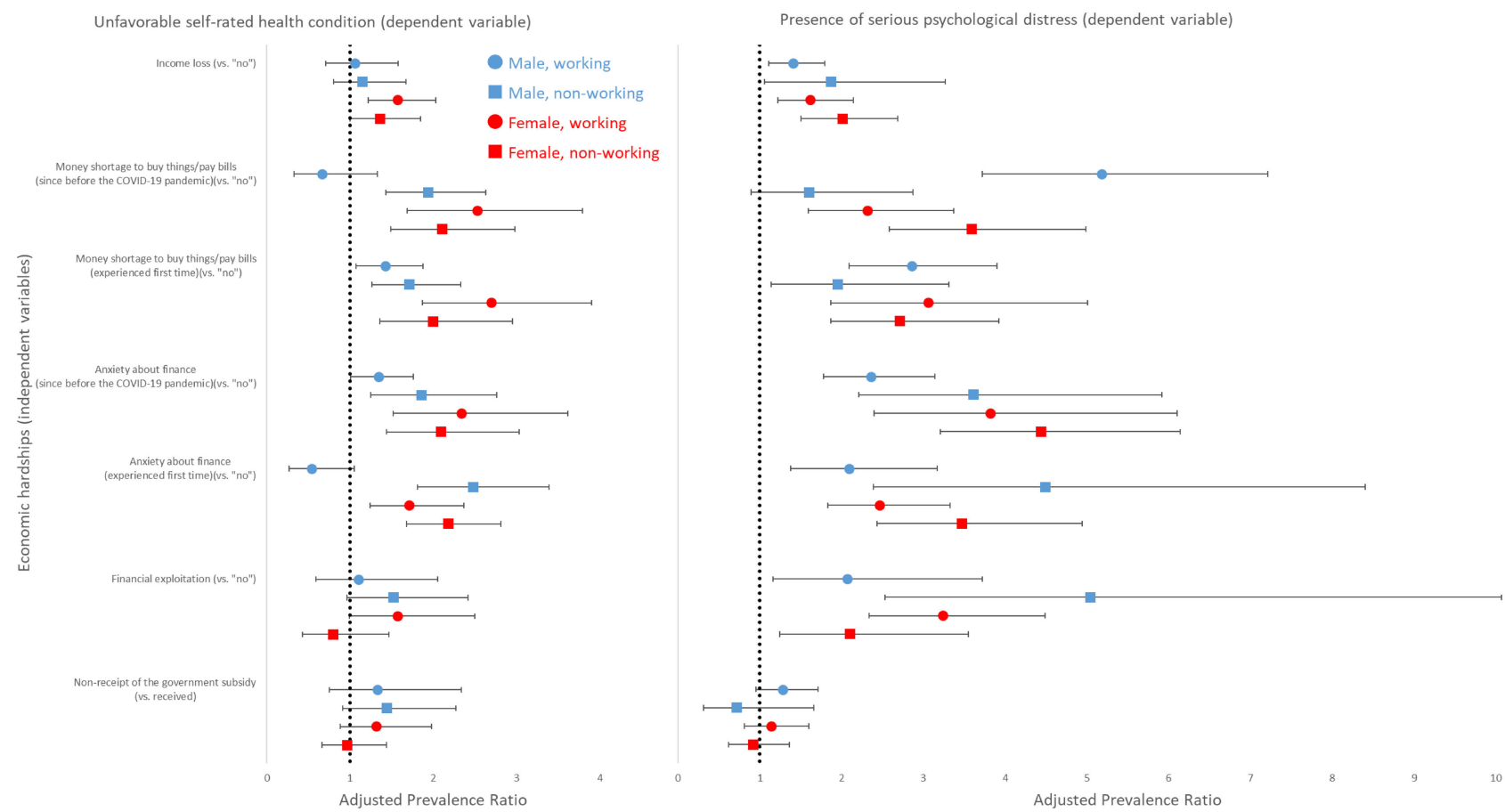

Figure 2. Associations between economic hardships and current state of health during the COVID-19 pandemic, 2020, Japan. $N=9,008$ (male, working); 3,665 (male, non-working); 6,446 (female, working); 6,363 (female, non-working). Genderand working status-specific adjusted prevalence ratios (95\% confidence intervals) estimated through weighted multivariable-adjusted log-linear models followed by robust variance estimation controlling for age, education, pastyear income level, marital status, cumulative COVID-19 cases in residing area, and history of physical and mental illness. Working status was categorized as to be working (full-time employee, self-employed, part-time/contractor) or non-working (retired, student, domestic worker, unemployed). Current health condition was defined as to be unfavorable (poor/fair) or favorable (excellent, very good, good) using a 5-point Likers scale. Presence of serious psychological distress was defined as scores $\geq 13$ using the Kessler-6 scale. COVID-19, coronavirus disease 2019.

income, younger and less affluent individuals were more likely to experience money shortage and financial anxiety during the COVID-19 pandemic. Furthermore, divorced or widowed individuals were nearly twice as likely to experience money shortage, despite their relatively low likelihood of income loss. These findings indicate that income loss during the COVID-19 pandemic have exaggerated the pre-existing economic inequalities by burdening the finance of various populations, and consequently, vulnerable individuals had to face material deprivation and financial anxiety.

Our analysis of financial exploitation and non-receipt of the cash relief provides important implications regarding personal or interpersonal experiences, especially those of non-working populations. We found that $13.6 \%$ of all respondents had not received the relief at the time of survey. As of August $27^{\text {th }}, 2020$, when our data collection was taking place, Japan's Ministry of Internal Affairs and Communications reported that the total program spending reached 12.59 trillion Japanese yen $(\$ 110$ billion in United States dollars), which had seemingly achieved $98.9 \%$ coverage. $^{10}$ It should be noted, however, that the relief program was administered on a household basis that allowed only the head of each household to file for and receive the relief on behalf of all household members. Therefore, the gap in nonreceipt percentage between our finding $(13.6 \%)$ and the official estimate $(1.1 \%)$ might be partially due to stagnated distribution within each household.

We further found similar patterns in non-receipt of the cash relief and financial exploitation: both were more common among male, younger, and divorced or widowed individuals. Due to the household-based administration, receipt of the cash relief could be affected by the power relationship within each household. It should also be noted that individuals with a history of physical and mental illness were more likely to experience financial exploitation, indicating that their financial autonomy may have been limited under the pandemic. These facts underscore the need to evaluate the COVID-19-related support programs from the public perspective to inform future interventions and maximize the reach to disadvantaged individuals.

Japan marked a drastic increase in suicide incidence since July 2020 , with the most prominent surge among young females. ${ }^{30,31}$ Recent assessments of Japanese workers have revealed a synchronized increase in psychological distress among younger females. ${ }^{16,17}$ Our study adds up to this evidence base by identifying the populations at higher risk of SPD and mental health deterioration including poorer individuals, divorced or widowed individuals, and those with a history of mental illness. We also found that individuals with a history of past or present mental illness were more likely to report physical health deterioration, possibly reflecting their limited resilience to cope with the physical or psychological stress imposed during the COVID-19 pandemic. Furthermore, exposure to financial exploitation was significantly associated with deterioration of physical and mental health among only non-working individuals, highlighting their vulnerability to the detrimental effects of such interpersonal experiences. Given that non-working population consists of the retired, students, and domestic workers, who might 
often lack social connectedness during or in the aftermath of the COVID-19 crisis, targeted interventions, such as remote counseling services and provision of information resources for individuals who seek help, are warranted.

Although we cannot infer causality from our cross-sectional data, our findings would suggest the bidirectional association between economic hardships and adverse health outcomes. Deterioration of physical and mental health was significantly higher among individuals who had been deprived of money or having financial anxiety since before the COVID-19 pandemic, suggesting that health outcomes were preceded by economic hardships. The opposite trajectory would also exist, given that individuals with a history of past morbidities were more likely to undergo economic hardships that arose during the pandemic. To address this bidirectional aspect of detrimental effects, implementation of social support programs to target the populations with heavier health burdens, in combination with safety net programs to mitigate the COVID-19-induced economic hardships, is necessary to prevent socioeconomic and health disparities from widening.

There are several limitations in the present study. First, due to the cross-sectional nature of the study, we were unable to establish the exact chronological mechanism of the association between exposure to economic hardships and health outcomes. Second, the self-reported nature of the survey might have resulted in misreport even after filtering out invalid responses. Third, as the study sample was collected through the internet-based recruitment, our findings may not be generalizable to the population with limited access or literacy to the internet. Nevertheless, such selection bias is considered to be minimal, as we used weighted data to address the differences in socioeconomic and demographic characteristics between respondents of the current internet survey and the nationally representative survey. Lastly, the limited sample size prevented us from nuanced analysis of economic hardships and health impacts that might have been experienced differently by type of industries that respondents belonged to.

\section{Conclusion}

During the COVID-19 pandemic, three components of economic hardship-income loss, shortage of money, and financial anxiety-were independently associated with deterioration of physical and mental health, unfavorable current general health condition, and presence of SPD. Financial exploitation predicted mental health deterioration among non-working populations. Timely and targeted interventions, in combination with detailed assessment of the health of the at-risk populations, are warranted to prevent both the socioeconomic and health disparities from widening under the COVID-19 crisis.

\section{ACKNOWLEDGEMENTS}

Funding: This study was funded by the Japan Society for the Promotion of Science (JSPS) KAKENHI Grants [grant number 17H03589; 19K10671; 19K10446; 18H03107; 18H03062; 21H04856], the JSPS Grant-in-Aid for Young Scientists [grant number 19K19439], Research Support Program to Apply the Wisdom of the University to tackle COVID-19 Related Emergency Problems, University of Tsukuba, and Health Labour Sciences Research Grant [grant number 19FA1005; 19FG2001]. Conflicts of interest: None reported.

\section{APPENDIX A. SUPPLEMENTARY DATA}

Supplementary data related to this article can be found at https:// doi.org/10.2188/jea.JE20210268.

\section{REFERENCES}

1. Ozili PK, Arun T. Spillover of COVID-19: impact on the Global Economy. Available at SSRN 3562570. 2020.

2. Paremoer L, Nandi S, Serag H, Baum F. Covid-19 pandemic and the social determinants of health. BMJ. 2021;372:n129.

3. GlobeScan. New Global Poll Documents the Pandemic's Impact on Inequality. GlobeScan. Accessed Feb 5, 2020. https://globescan. com/global-poll-pandemic-impact-inequality/.

4. Witteveen D. Sociodemographic inequality in exposure to COVID19-induced economic hardship in the United Kingdom. Res Soc Stratif Mobil. 2020;69:100551.

5. Despard M, Grinstein-Weiss M, Chun Y, Roll S. COVID-19 job and income loss leading to more hunger and financial hardship. Washington, DC: Brookings Institution; 2020.

6. Ku L, Brantley E. Widening social and health inequalities during the COVID-19 pandemic. American Medical Association. JAMA Health Forum. 2020;1(6):e200721.

7. U.S. Internal Revenue Service. Economic Impact Payments. U.S. Internal Revenue Service. Accessed Feb 5, 2021. https://www.irs. gov/coronavirus/economic-impact-payments.

8. Korea Ministry of Economy and Finance. Comprehensive Economic Policy Response to the COVID-19 Pandemic. Korea Ministry of Economy and Finance. Accessed Feb 5, 2021. https://english.moef. go.kr/popup/20200417_policyFocus/popup.html.

9. BBC News. Coronavirus bailouts: Which country has the most generous deal? BBC News. Accessed Feb 5, 2021. https://www. bbc.com/news/business-52450958.

10. Japan Ministry of Internal Affairs and Communications. Special Cash Payment. Japan Ministry of Internal Affairs and Communications. Accessed Feb 5, 2021. https://www.soumu.go.jp/menu_ seisaku/gyoumukanri_sonota/covid-19/kyufukin.html.

11. Japan Ministry of Internal Affairs and Communications. Guide to Special Cash Payment. Japan Ministry of Internal Affairs and Communications. Accessed February 8, 2021. https://www.soumu. go.jp/main_content/000715668.pdf.

12. Ikeda T, Igarashi A, Odani S, Murakami M, Tabuchi T. Healthrelated quality of life during COVID-19 pandemic: assessing impacts of job loss and financial support programs in Japan. Appl Res Qual Life. 2021:1-17.

13. Dorn AV, Cooney RE, Sabin ML. COVID-19 exacerbating inequalities in the US. Lancet. 2020;395(10232):1243-1244.

14. Nicola O, Scott R. Economic hardship, health and COVID-19. Institute and Faculty of Actuaries. Accessed February 5, 2021. https://www.actuaries.org.uk/system/files/field/document/Covid19_ EconomicStressHealth\%20v1.2_clean.pdf.

15. Witteveen D, Velthorst E. Economic hardship and mental health complaints during COVID-19. Proc Natl Acad Sci USA. 2020; 117(44):27277-27284.

16. Sasaki N, Kuroda R, Tsuno K, Kawakami N. The deterioration of mental health among healthcare workers during the COVID-19 outbreak: a population-based cohort study of workers in Japan. Scand J Work Environ Health. 2020;46(6):639-644.

17. Sasaki N, Kuroda R, Tsuno K, Imamura K, Kawakami N. Deterioration in mental health under repeated COVID-19 outbreaks greatest in the less educated: a cohort study of Japanese employees. J Epidemiol. 2021;31(1):93-96.

18. Kikuchi H, Machida M, Nakamura I, et al. Changes in psychological distress during the COVID-19 pandemic in Japan: a longitudinal study. J Epidemiol. 2020;30(11):522-528.

19. Arber S, Fenn K, Meadows R. Subjective financial well-being, income and health inequalities in mid and later life in Britain. Soc Sci Med. 2014;100:12-20.

20. Guio AC, Fusco A, Marlier E. A European Union approach to 
material deprivation using EU-SILC and Eurobarometer data. 2009.

21. Rakuten Insight Inc. About Us: Rakuten Insight. Rakuten Insight Inc. Accessed Feb 22, 2021. https://insight.rakuten.co.jp/en/aboutus. html.

22. Tabuchi T, Shinozaki T, Kunugita N, Nakamura M, Tsuji I. Study profile: The Japan "Society and New Tobacco" Internet Survey (JASTIS): a longitudinal internet cohort study of heat-not-burn tobacco products, electronic cigarettes, and conventional tobacco products in Japan. J Epidemiol. 2019;29(11):444-450.

23. Schenck-Fontaine A, Panico L. Many kinds of poverty: three dimensions of economic hardship, their combinations, and children's behavior problems. Demography. 2019;56(6):2279-2305.

24. Kessler RC, Andrews G, Colpe LJ, et al. Short screening scales to monitor population prevalences and trends in non-specific psychological distress. Psychol Med. 2002;32(6):959-976.

25. Japan Ministry of Health, Labour and Welfare. Overview of the 2018 Comprehensive Survey of Living Condition of the People on Health and Welfare. Japan Ministry of Health, Labour and Welfare. Accessed Feb 9, 2021. https://www.mhlw.go.jp/toukei/saikin/hw/ k-tyosa/k-tyosa18/index.html.
26. JAG Japan. Coronavirus COVID-19 Japan case by each prefecture (2019-nCoV). JAG Japan. Accessed Feb 9, 2021 https://jagjapan.maps.arcgis.com/apps/opsdashboard/index.html\#/ $55 \mathrm{c} 22 \mathrm{ee} 976 \mathrm{bc} 42338 \mathrm{cb} 454765 \mathrm{a} 6 \mathrm{edf} 6 \mathrm{~b}$

27. Japan Ministry of Health, Labour and Welfare. https://www.mhlw. go.jp/english/database/db-hss/cslc.html. Japan Ministry of Health, Labour and Welfare. Accessed April 9, 2021. https://www.mhlw. go.jp/english/database/db-hss/cslc.html.

28. Tabuchi T, Kiyohara K, Hoshino T, Bekki K, Inaba Y, Kunugita N. Awareness and use of electronic cigarettes and heat-not-burn tobacco products in Japan. Addiction. 2016;111(4):706-713.

29. Statistics Bureau of Japan, Ministry of Internal Affairs and Communications. Labour Force Survey. Statistics Bureau of Japan, Ministry of Internal Affairs and Communications. Accessed Feb 5, 2021. https://www.stat.go.jp/english/data/roudou/index.html.

30. Ueda M, Nordström R, Matsubayashi T. Suicide and mental health during the COVID-19 pandemic in Japan. medRxiv. 2020.

31. Japan National Police Agency. Statistics: suicide. Japan National Police Agency. Accessed Feb 8, 2021. https://www.npa.go.jp/ publications/statistics/safetylife/jisatsu.html. 